\title{
Open Science und Citizen Science als symbiotische Beziehung?
}

\author{
Eine Gegenüberstellung von Konzepten
}

\section{8}

In diesem Artikel werden Kernzuschreibungen von Open Science und Citizen Science in der europäischen Wissenschaftspolitik analysiert und zueinander in Beziehung gesetzt. Beide Konzepte postulieren und forcieren eine zukünftige Öffnung von Wissenschaft - wobei die Open-Science-Politik vor allem auf den Prozess von Wissenschaft abhebt, während bei der Konzeptualisierung von Citizen Science die Akteure und ihre Interaktionen stärker im Vordergrund stehen. Beiden Ansätzen gemeinsam ist der Anspruch an einen transparenten Forschungsprozess und frei zugängliche Forschungsergebnisse. Es gibt Synergien, was die Einbindung von Bürgerinnen und Bürger sowie die Zugänglichkeit von Forschungsergebnissen und -prozessen angeht. Risiken bestehen, wenn Citizen Science lediglich instrumentalisiert wird, ohne Bürgerinnen und Bürgern eine echte Teilhabe zu ermöglichen.

\section{Open Science and Citizen Science - a symbiotic relationship? A juxtaposition of core concepts}

This article analyzes core attributes of the concepts of Open Science and Citizen Science in European science policy and relates them to one another. Both concepts postulate and advocate a future opening of science - with Open Science policy focusing on the research process and the conceptualization of Citizen Science placing more emphasis on the actors and their interactions. Both approaches claim transparency with regard to both the research process itself and its results. There are synergies in terms of the involvement of citizens and the accessibility of research results and processes. And there are risks when citizen Science is merely instrumentalized without allowing for effective participation by citizens.

KEYWORDS: open science, citizen science, public engagement in science and technology

This is an article distributed under the terms of the Creative Commons Attribution License CCBY 4.0 (https://creativecommons.org/licenses/by/4.0/)

https://doi.org/10.14512/tatup.26.1-2.18

Eingereicht: 15. 03.2017. Angenommen: 02.06.2017

\section{Einleitung}

Open Science (offene Wissenschaft) und Citizen Science (Bürgerwissenschaft oder Bürgerforschung) geben das große Versprechen, gemeinsam Barrieren zwischen Wissenschaft und Gesellschaft aufzuheben. Eine Öffnung der Wissenschaft, die schon länger als Begleiterscheinung der Digitalisierung von Wissenschaft beobachtet wird (Nentwich und König 2012), wird mit der Open-Science-Strategie als Forderung in der europäischen forschungspolitischen Agenda verankert. Nun ist Citizen Science zu einer der wichtigen Säulen in der konzeptionellen Darstellung von Open Science geworden (European Commission 2016b). Gleichzeitig werden die Möglichkeiten von Digitalisierung und Open Science als Chance für Citizen-Science-Aktivitäten wahrgenommen (Newman et al. 2012) und zunehmend Thema in der Citizen-Science-Community, z. B. in der European Citizen Science Association (https://ecsa.citizen-science.net/). Die Konzepte von Citizen Science und Open Science stellen somit Bezugspunkte in aktuellen forschungspolitischen Diskursen dar, welche sich zunehmend aufeinander beziehen.

Der vorliegende Beitrag nimmt die aktuellen Konkretisierungsbemühungen der Open-Science-Politik auf europäischer Ebene zum Anlass, um mit dem Fokus auf Citizen Science tragende Begriffe und Argumentationslinien aus beiden Diskursen zu rekonstruieren und auf mögliche Anknüpfungspunkte hin zu befragen. Dies geschieht zum einen mit dem Ziel, aus dem Praxiskontext der Bürgerwissenschaften heraus Ausgangspunkte für weiterführende Diskussionen mit Vertretern der Wissenschaftsund Technikforschung aufzuzeigen. Zum anderen hat dieser Beitrag den Anspruch, konstruktive Impulse für kritische Reflexionen in diesen Praxiskontext zurück zu spiegeln.

\section{Methode}

Zur Analyse der Argumentationslinien von Open Science und Citizen Science auf europäischer Ebene wurde eine Dokumentenanalyse von zentralen Strategiepapieren der EU-Kommission und 


\begin{tabular}{|l|l|l|}
\hline Typ & Open Science & Citizen Science \\
\hline $\begin{array}{l}\text { Zentrale politische Strategiepapiere auf } \\
\text { europäischer Ebene }\end{array}$ & $\begin{array}{l}\text { Programmatische Papiere der Europäischen Kommis- } \\
\text { sion (Moedas 2016; European Commission 2016 b) }\end{array}$ & $\begin{array}{l}\text { White Paper im Auftrag der Europäischen Kommission } \\
\text { (Sanz et al. 2014) }\end{array}$ \\
\hline $\begin{array}{l}\text { Wissenschaftliche Publikationen zu } \\
\text { Hauptdiskursen }\end{array}$ & $\begin{array}{l}\text { Review zu Open-Science-Strömungen } \\
\text { (Friesike et al. 2015) } \\
\text { Open Science als Demokratisierungsbewegung } \\
\text { (Dickel und Franzen 2015) } \\
\text { Crowd Science (Franzoni und Sauermann 2014) }\end{array}$ & $\begin{array}{l}\text { Bibliometrische Publikationsanalyse } \\
\text { (Kullenberg und Kasperowski 2016) } \\
\text { Hauptreferenzen zum Grundverständnis } \\
\text { (Bonney et al. 2009; Shirk und Bonney 2015; } \\
\text { Irwin 1995) }\end{array}$ \\
\hline $\begin{array}{l}\text { Definitionen guter Praxis aus der Zivil- } \\
\text { gesellschaft }\end{array}$ & $\begin{array}{l}\text { Open Definition 2.1 von Open Knowledge International } \\
\text { (Open Knowledge International 2015) }\end{array}$ & $\begin{array}{l}\text { 10 Prinzipen guter Praxis in Citizen Science } \\
\text { (ECSA 2015) }\end{array}$ \\
\hline
\end{tabular}

Tab.1: Gruppierung der zur Analyse herangezogenen Kernpublikationen.

Quelle: Eigene Darstellung

Best-Practice-Dokumenten zivilgesellschaftlicher Dachverbände durchgeführt und durch Forschungsliteratur zu wichtigen Bestandteilen der jeweiligen Diskussionen ergänzt (Tab. 1). Da beide Konzepte vielstimmigen aktuellen Diskussionen entspringen, muss eine Positionsbestimmung partiell bleiben. Wo möglich, wird auf weniger prominente Debatten und Akteure verwiesen.

Der europäische Open-Science-Diskurs wird v. a. auf der Ebene der Wissenschaftspolitik in Interaktion mit akademischen Forschungscommunities vorangetrieben. So wurde das Open Science Policy Panel (OSPP) als Beratungsgremium für die EU-Kommission einberufen, in welchem die Vertreter großer Forschungseinrichtungen und -infrastrukturen, der Wirtschaft und der European Citizen Science Association (ECSA) vertreten sind, allerdings (noch?) nicht die entsprechenden zivilgesellschaftlichen Initiativen wie z. B. die Open Knowledge Foundation oder Wikimedia (European Commission 2016a). Demgegenüber ist der Citizen-Science-Diskurs weit weniger eindeutig zuordenbar. So besteht die Citizen-Science-Community, wenn überhaupt von einer Community gesprochen werden kann (Kullenberg und Kasperowski 2016), aus den verschiedensten akademischen und zivilgesellschaftlichen Gruppen, welche Ansätze partizipativer Forschung praktizieren.

Dies spiegelt sich auch in der Verfügbarkeit von Konzeptpapieren wider. Während im Bereich Open Science Strategiepapiere auf europäischer Ebene zu finden waren, gab es hierzu kaum Dokumente von zivilgesellschaftlichen Initiativen. Bei Citizen Science ist es umgekehrt: Die offiziellen Papiere sind eher wissenschaftliche Zustandsbeschreibungen des Feldes oder wurden, wie beispielsweise beim Socientize-Projekt (Sanz et al. 2014), von der EU-Kommission beauftragt. Selbstzuschreibungen aus den jeweiligen zivilgesellschaftlichen Communities mit übergreifendem Charakter finden sich nur bei der ECSA.

Basierend auf diesen Papieren wurde analysiert, welche Konzepte sich hinter den Schlagwörtern versammeln, und wie diese aufeinander bezogen sind. Anhand von einschlägigen Kernzuschreibungen als Vergleichsdimensionen werden mögliche Synergien sowie Zielkonflikte diskutiert und daraus strategische Kooperationsfelder abgeleitet.

\section{Kernkonzepte und gegenseitige Bezugnahmen}

Open Science (offene Wissenschaft) ist ein Überbegriff, dessen intuitive umgangssprachliche Ebene - ,jeder kann mitmachen, alles liegt offen vor" im Sinne von offenem Wissen (Open Knowledge International 2015) - nicht identisch mit dem technischen Begriff Open Science ist, der sich auf den Wissenschaftsprozess bezieht und damit nicht voraussetzungsfrei ist. Zum einen bezeichnet Open Science ein neues Paradigma in der Art, wie Forschung betrieben wird (Friesike et al. 2015). Dabei steht nicht nur die Zugänglichkeit von Daten und Methoden, sondern vor allem die frühzeitige Öffnung des Forschungsprozesses im Mittelpunkt. Zum anderen stellt Open Science eine Strategie dar, mittels Digitalisierungsmaßnahmen Wissenschaft effizienter zu gestalten (Franzen 2016). Auch die Beteiligung von Nicht-Wissenschaftlerinnen an Forschungs- und Wissenschaftskommunikationsprozessen wird als wesentlicher Teil von Open Science angesehen (Fecher und Friesike 2014).

Der Begriff Citizen Science hat zwei komplementäre Ursprünge. Zum einen speist er sich aus der naturkundlichen Tradition von ehrenamtlichen Forscherinnen und Forschern, deren Daten Eingang in das wissenschaftliche System finden (Bonney 1996), wobei Wissenschafts- und Umweltbildung als zentraler Vorteil für die Beteiligten angesehen wird (Bonney et al. 2009). Zum anderen wurde Citizen Science zeitgleich als emanzipatorischer Ansatz zur Mobilisierung alternativer Formen von Expertise im Kontext von Umweltpolitik thematisiert (Irwin 1995). Im Folgenden werden die zentralen Begriffe - Open Science und Citizen Science - aus Sicht der europäischen Wissenschaftspolitik und aus Perspektive der europäischen Citizen-Science-Community rekonstruiert.

\section{Open Science aus der Sicht der EU-Wissenschaftspolitik}

Im Kontext der EU-Wissenschaftspolitik adressiert Open Science in erster Linie veränderte Möglichkeiten des wissenschaftlichen Arbeitens durch die digitale Revolution und diesbezüg- 
lich ausgemachte Regulierungsbedarfe. Durch die enge Verbindung zu den Bereichen Open Innovation und Open to the World in der sogenannten ,3Os“-Strategie (European Commission 2016 b) wird die Forschungspolitik der Kommission klar im Kontext von Wirtschaftsförderung und internationaler Wettbewerbsfähigkeit positioniert.

Hauptdebatten betreffen erstens Zugang zu und Nutzungsrechte an Publikationen und Daten, Stichworte Open Access und Open Data (Friesike et al. 2015), und damit verbundene Verschiebungen im Publikationswesen. Zweitens werden technische Voraussetzungen anhand der Open-Science-Cloud diskutiert, eine große technische Infrastruktur für wissenschaftliche Einrichtungen zur Bearbeitung großer Datenmengen (Grossman et al. 2012). Ein dritter Fokus liegt auf Anreizsystemen für Forschende durch entsprechende Leistungsmessung, insbesondere die als altmetrics bezeichneten Metriken für Artikelzitationen und Nutzungsnachweise anderer Ergebnisse wissenschaftlicher Arbeit wie Daten, Software oder Blogposts (Holmberg 2015).

\section{Unterstützungsleistungen in der Datenanalyse}

,[J]ust as people offer spare rooms via AirBnB, why shouldn't they be allowed to offer spare brain power via citizen science?" (European Commission 2016b, S. 34)

Zum anderen werden Projekte hervorgehoben, welche auf die massenhafte Klassifikation von Daten setzen, z. B. durch sogenanntes Taggen oder indem Teilnehmende Bilder oder Videos beschreiben, die dann als Metadaten verfügbar sind. Als ein Beispiel wird die internationale Plattform Zooniverse genannt, die einen Zugang zu dieser Art von Projekten bietet. Hier liegt der Beitrag der Teilnehmenden in erster Linie in der Mustererkennung und dient als Grundlage für eine maschinelle Weiterbearbeitung.

3. Wissenschaftsfreundlichkeit und Anregungen aus der Gesellschaft

„Citizen Science is often linked with outreach activities, science education or various forms of public engagement with science as a way to promote Responsible Research and Innovation." (European Commission 2016b, S. 54)

\section{Open Science zielt auf eine Öffnung durch Technik hin zu einer datengetriebenen Wissenschaft, Citizen Science bezieht sich in erster Linie auf Beteiligung.}

\section{Citizen Science in der EU-Open-Science-Politik}

Eine Neuerung in der Open-Science-Diskussion auf EU-Ebene stellt die verstärkte Thematisierung von Citizen Science dar. Als Teil der Politiklinie „Fostering and creating incentives for Open Science“" wird Bürgerwissenschaft generell als Möglichkeitsraum für die Erschließung zusätzlicher Ressourcen für Forschung gefasst. Bezüge zu Citizen Science im ,3 Os“ Konzept sprechen drei Aspekte an:

\section{Unterstützungsleistungen in der Datenaufnahme}

,[I]f scientists want to monitor the effects of climate change on local ecosystems, they can use Open Science to engage citizen reporting, and rapidly multiply the data at their disposal.“ (European Commission 2016b, S. 35)

Zum einen verspricht die Beteiligung der breiten Öffentlichkeit an Forschungsprojekten Forschungsdaten, die bisher unzugänglich für Wissenschaftler sind, und damit eine Verbesserung von Datenbasis und -qualität. Dabei wird Citizen Science als verteilte Community mit kleineren und einfacheren Datensätzen im „long tail of science“ verortet, und als Gegenpol zu zentralisierten Fachcommunities, die mit Big Data und großen Infrastrukturen operieren, verstanden (European Commission 2016 b, S.47). Als zentrales Beispiel dienen die von der EU-Kommission geförderten Citizen-Observatory-Projekte (http://citizen-obs.eu/) im Anwendungsfeld der Fernerkundung.
Neben den oben beschriebenen zwei konkreten Arten von Zuarbeit zu Forschungsprojekten wird Citizen Science außerdem als Form von Wissenschaftskommunikation und -bildung ausgewiesen. Im Sinne einer breiter und aktiver verstandenen Auseinandersetzung mit Wissenschaft soll ein Beitrag $\mathrm{zu}$ verantwortlicher Forschung (im Sinne von Responsible Research and Innovation - RRI) geleistet und deren gesellschaftliche Relevanz verbessert werden (European Commission 2013). In diesem Kontext wird auch auf unabhängige Do-it-Yourself-Forschung (DIY) verwiesen. Als Beispiel für diese vielgestaltige und wachsende Szene dient Genspace, eine NGO mit eigenem Biologielabor in New York.

Mit Citizen Science soll die datengetriebene Forschung einen verlängerten Arm erhalten, der in die Zivilgesellschaft hineinreicht. Partizipation seitens der breiten Öffentlichkeit soll in Wert gesetzt werden, um Innovationskraft und Wettbewerbsfähigkeit zu erhöhen. Sie dient entweder als Hilfsarbeit für Forschung - in der Form von Datengewinnung und -analyse in akademisch-initiierten Projekten, was eher dem Konzept von Crowdsourcing oder Crowd Science (Franzoni und Sauermann 2014) entspricht; oder Partizipation wird gefasst als Bildungsund Engagementaktivität im gesellschaftlichen Kontext von Wissenschaft, die Akzeptanz für Forschung sichert und auch gelegentliche Reizungen durch Graswurzelbewegungen tolerieren soll. 


\begin{tabular}{|l|l|}
\hline Definition & Quelle \\
\hline $\begin{array}{l}\text { Citizen Science refers to the general public engagement in scientific research activities when citizens } \\
\text { actively contribute to science either with their intellectual effort or surrounding knowledge or with their tools } \\
\text { and resources. }\end{array}$ & Sanz et al. (2014) \\
\hline $\begin{array}{l}\text { [Environmental] citizen science encompasses many different ways in which citizens are involved in science. } \\
\text { This may include mass participation schemes in which citizens use smartphone apps to submit wildlife } \\
\text { monitoring data, as well as smaller-scale activities, for example, grassroots groups taking part in local policy } \\
\text { debates about fracking. }\end{array}$ & Science Communication Unit (2013) \\
\hline $\begin{array}{l}\text { The collection and analysis of data relating to the natural world by members of the general public, typically } \\
\text { as part of a collaborative project with professional scientists. }\end{array}$ & Oxford Dictionary (2017) \\
\hline $\begin{array}{l}\text { Citizen science is defined as organised research in which the balance between scientific, educational, societal } \\
\text { and policy goals varies across projects. }\end{array}$ & European Citizen Science Association (2017) \\
\hline
\end{tabular}

\section{Citizen Science aus der Innensicht}

In den Reihen von Citizen-Science-Projektorganisatoren und deren Verbänden sind in den letzten Jahren verschiedene Definitionen von Citizen Science entstanden, die sich konzeptionell zum Teil stark unterscheiden und sehr unterschiedlich breit ausgelegt werden (Tab. 2). Zwei entscheidende Merkmale, grob als Forschung und Partizipation umschrieben, sind ihnen gemeinsam.

Das erste Merkmal: Citizen Science beschreibt originäre Forschung, Citizen-Science-Aktivitäten tragen zum wissenschaftlichen Erkenntnisgewinn bei. In welchem Teil des Forschungsprozesses Bürgerinnen eingebunden werden - ob bei der Formulierung von Forschungsfragen, Entwicklung von Methoden, Erhebung und Auswertung von Daten oder Interpretation der Ergebnisse - unterscheidet sich zwischen den Akteuren. Einige sehen Bürger vor allem in der Rolle von Datenlieferanten, andere rechnen auch Projekte dazu, in denen Bürgerinnen und Bürger Daten auswerten oder die Fragestellung (mit-)entwickeln. Die European Citizen Science Association (2015) bietet mit den zehn Prinzipien zu Citizen Science einen Katalog von Merkmalen guter Praxis an, der in zweijähriger Konsultation von Praktikerinnen und Praktikern ausgearbeitet wurde. Darin wird nahegelegt, Bürgerinnen und Bürger über die Datenerhebung hinaus am Forschungsprozess zu beteiligen. Noch in der Diskussion ist, inwieweit der Begriff Citizen Science für Geistes- und Sozialwissenschaften greift (Pettibone et al. 2016). Teilweise gibt es dort Überschneidungen mit anderen Forschungstraditionen wie „partizipativer Aktionsforschung“ oder „Co-Design“.

Das zweite übergreifende Merkmal von Citizen-ScienceDefinitionen aus dem Feld betrifft die mit der Beteiligung an Forschung verbundenen Motivationen und Ziele: Bürgerinnen und Bürger engagieren sich aus eigenem Interesse und selbstbestimmt an der Forschung und sind dafür nicht angestellt. Die Ziele von Citizen-Science-Projekten sind vielfältig - es kann um Naturschutz, Lokalgeschichte oder Astronomie gehen; die Stärkung der Zivilgesellschaft kann ebenso eine Rolle spielen wie die Erweiterung der wissenschaftlichen Datenbasis (Irwin 1995; Kullenberg und Kasperowski 2016). Auch die Beteilig- ten selbst haben sehr diverse Vorstellungen (Pettibone et al. in Vorbereitung). Citizen Science ist somit mit fast so vielen Visionen von Wissenschaft und Gesellschaft verbunden, wie es Initiativen gibt.

\section{Open Science aus Sicht der Citizen-Science- Community}

Der Begriff Open Science ist innerhalb der Citizen-ScienceCommunity nicht weit verbreitet und nur vereinzelt finden sich explizite Referenzen, z. B. bei Veranstaltungen in Kooperation mit der Open Knowledge Foundation'. Auch die ECSA-Arbeitsgruppe zum Thema Open Science wurde erst Anfang 2017 gegründet. Ausnahmen bilden DIY-Communities, welche sich für freie Lizenzen in der Molekularbiologie einsetzen (Trojok 2016) oder offene Hardware für Umweltbeobachtungen entwickeln, wie das Public Laboratory of Open Technology (Preston 2016).

\section{Diskussion}

Auf der Ebene der Konzepte finden sich in Open Science und Citizen Science zwei Zeitdiagnosen über Wissenschaft, die aufbauend auf unterschiedlichen Diskursen gegenwärtige Veränderungen im Wissenschaftssystem beschreiben und Visionen für dessen zukünftige Gestalt entwerfen. Dabei stellt das Narrativ von Open Science digitale Kommunikationstechnologien in den Vordergrund - beschrieben wird eine Öffnung durch Technik hin zu einer datengetriebenen Wissenschaft, die effektiver ist und deren Zwischenschritte zugänglicher sind als bisher. Demgegenüber bezieht sich das Narrativ von Citizen Science in erster Linie auf Beteiligung. Angestrebt wird eine Öffnung für die

1 Zwei Beispiele sind eine Abendveranstaltung zum Thema Open Science und BürgerInnenwissenschaft in Österreich (http://www.wtz-ost.at/veranstaltungen/ open-science-und-buerger_innenwissenschaft) und ein Projekt von Open Knowledge Finnland (https://fi.okfn.org/projects/open-citizen-science). 


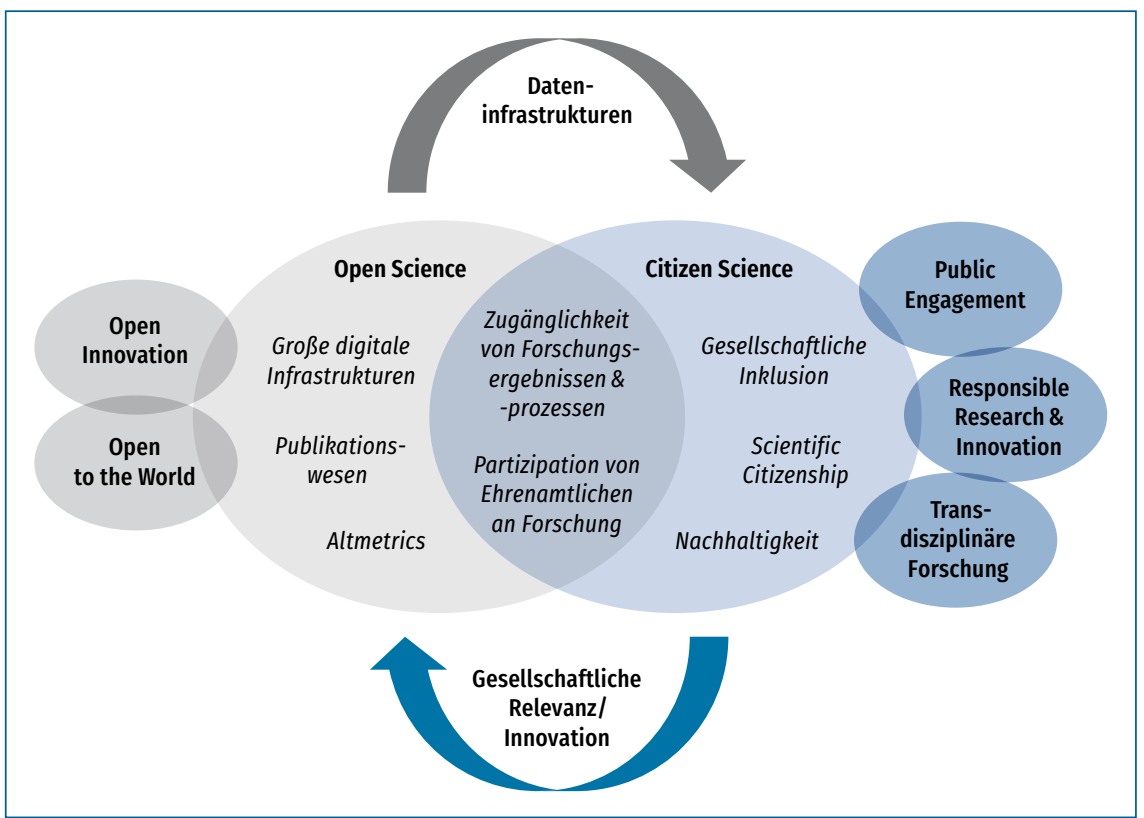

Abb.1: Open-Science- und Citizen-Science-Kernkonzepte und Anknüpfungspunkte.

Quelle: Eigene Darstellung auf Massen unbezahlter Hilfskräfte andererseits kritisch zu betrachten. Um Citizen Science besser im Open-Science-Diskurs zu verankern, bedarf es eines breiteren Spektrums an Partizipation, d. h. BürgerwissenschaftlerInnen werden nicht darauf beschränkt, der Wissenschaft zuzuarbeiten oder Forschungsergebnisse zu rezipieren.

\section{Wie offen ist Citizen Science?}

Oben wurde angesprochen, dass sich obwohl nicht explizit mit Open Science in Verbindung gebracht - zahlreiche Anknüpfungspunkte innerhalb der CitizenScience-Community bezüglich Zugänglichkeit von Forschungsdaten und -prozessen finden. Allen voran gilt das für das Thema offene Daten sowie der damit verbundenen Möglichkeit der erweiterten Zusammenarbeit und Mobilisierung von Bevölkerungsgruppen (Newman et al. 2012). Allerdings wird der Citizen-Sci-

verschiedensten Formen von Partizipation von Ehrenamtlichen an Forschungsprozessen mit diversen Zielen, u. a. der Generierung von Forschungsdaten und einer robusteren Wissenschaft.

\section{Wie inklusiv ist Open Science?}

In den Strategiepapieren zu Open Science wird Citizen Science als Mechanismus für eine inklusivere Wissenschaft verstanden. Partizipation dient dem Zweck, Arbeitskraft und Daten für konkrete Forschungsprozesse sowie gesellschaftliche Akzeptanz für Forschung durch Wissenschaftskommunikation und -bildung zu mobilisieren. Wer sich beteiligt, wird nicht weiter spezifiziert man geht von einer anonymen breiten Öffentlichkeit, als crowd

\section{Wesentlich für Citizen Science ist, dass sich Qualität nicht nur auf}

\section{die Daten, sondern auch auf die Art der Zusammenarbeit bezieht.}

verstanden, aus. Das Muster für partizipative Projekte ist klar wissenschaftsgetrieben (European Commission 2016b, S. 53). Diese Vorstellung zeigt sich auch auf der Ebene der Indikatoren. Für Open Science sind bisher lediglich Indikatoren zu Publikationsmodi und Datenrepositorien im Gespräch (Smith et al. 2016).

Vor diesem Hintergrund ist das im Open-Science-Konzept auftretende Verständnis von Citizen Science als Platzhalter für Public Engagement with Science einerseits und als Reduktion
ence-Community selbst eine unzureichende Praxis der Zur-Verfügung-Stellung von Daten bescheinigt, insbesondere was den Bereich der Biodiversitätsdaten angeht (Groom et al. 2016).

Gegenwärtige Initiativen zum Thema Interoperabilität umfassen die Erarbeitung internationaler Standards für Projekt und Beobachtungsdaten (Schade et al. 2016) sowie Leitlinien für die Nachnutzung von Citizen-Science-Apps und Online-Plattformen (Luna und Sturm 2017).

Zusammen mit den weiter oben angesprochenen Initiativen zu offener Hardware und freien Lizenzen finden sich im Feld der Citizen Science eine Reihe von Anknüpfungspunkten beim Thema Zugänglichkeit von Daten und Forschungsprozessen. Um hier anzudocken, empfiehlt sich eine Schärfung von Open-Science-Konzepten für disziplin- bzw. fachkulturspezifische Unterschiede an informationstechnischen Bedarfen und Umgängen.

\section{Fazit}

Der Vergleich von Open Science und Citizen Science steht vor der konzeptionellen Herausforderung, dass es sich zum einen vor allem um eine europäische Strategie für die Öffnung von Wissenschaft handelt und zum anderen um eine gelebte partizipative Forschungspraxis. Entsprechend finden die Diskurse teilweise auf unterschiedlichen Ebenen statt. Anknüpfungspunkte zwischen Open Science und Citizen Science ergeben sich zum einen auf der Ebene der Zugänglichkeit von Forschungsergebnissen und -prozessen, zum anderen auf der Ebene der Partizipation (Abb. 1). Die Citizen-Science-Praxis kann stark von der Öffnung des Wissenschaftssystems profitieren, insbesondere von der besseren Zugänglichkeit zu Daten, Informationen und 
Werkzeugen. Für eine Umsetzung von Aktivitäten in diesem Schnittfeld sind erweiterte Anreizsysteme und die Bedarfe der unterschiedlich verfassten Communities einzubeziehen. Wesentlicher für Citizen Science aber ist, dass die Möglichkeit der Beteiligung erweitert wird und sich Qualität nicht nur auf die Daten, sondern auch auf die Art der Zusammenarbeit bezieht. Ressourcen werden nicht nur benötigt, um die entsprechenden Infrastrukturen auszubauen, sondern auch, um Kommunikation und Inklusion im Hinblick auf verschiedene soziale Gruppen und ihrer Interessen in der Gesellschaft zu fördern. Hier können die Open-Science-Strategen von den Citizen-Science-Communities lernen und Bürgerinnen und Bürger nicht nur als Zulieferer von Daten betrachten, sondern stärker als Treiber von Innovation im Hinblick auf einen Beitrag zum großen Ganzen: einer gesellschaftlichen Transformation hin zu mehr Nachhaltigkeit. In diesem Zusammenhang ist auch mehr Austausch zwischen akademischer Wissenschafts- und Technikforschung und den Praxiskontexten der Citizen Science wünschenswert (Mahr et al. im Druck), um kritische Reflexion stärker in den jeweiligen Diskursen zu verankern, von Forschungsergebnissen aus anderen Praxisfeldern zu profitieren und zukünftige Entwicklungen verantwortlich zu gestalten.

\section{Danksagung}

Wir bedanken uns bei den Kolleginnen und Kollegen der Abteilung „Wissenschaft in der Gesellschaft - WiG" des Museums für Naturkunde Berlin für die konstruktive Diskussion des Manuskriptentwurfs, namentlich Susanne Schmitt, Katharina Wischmeyer, Julia Wiedemann, Julia Diekämper, André Mascarenhas, Maike Fischer, Soledad Luna und David Ziegler.

\section{Literatur}

Bonney, Rick (1996): Citizen Science: A Lab Tradition. In: Living Bird 15 (4), S. 7-15. Bonney, Rick et al. (2009): Citizen Science: A Developing Tool for Expanding Science Knowledge and Scientific Literacy. In: BioScience 59 (11), S. $977-984$. DOI: 10.1525/bio.2009.59.11.9.

Dickel, Sascha; Franzen, Martina (2015): Digitale Inklusion: Zur sozialen Öffnung des Wissenschaftssystems. In: Zeitschrift für Soziologie 44 (59), S. 330-347.

ECSA - European Citizen Science Association (2015): 10 Principles of Citizen

Science. Online verfügbar unter http://ecsa.citizen-science.net/documents, zuletzt geprüft am 03.03.2017.

ECSA - European Citizen Science Association (2017): About Us. Online verfügbar unter https://ecsa.citizen-science.net/about-us, zuletzt geprüft am 09. 06.2017.

European Commission (2013): Responsible Research and Innovation: Options for Strengthening Research and Innovation. Report of the Expert Group on the State of Art in Europe on Responsible Research and Innovation. Onliner verfügbar unter https://ec.europa.eu/research/science-society/ document_library/pdf_06/options-for-strengthening_en.pdf, zuletzt geprüft am 09. 06.2017.

European Commission (2016a): Nominated Members of the Open Science Policy Platform. Online verfügbar unter http://ec.europa.eu/research/openscience/ pdf/ospp_nominated_members.pdf, zuletzt geprüft am 09.06.2017.

European Commission (2016 b): Open Innovation, Open Science, Open to the World - a Vision for Europe. Directorate-General for Research and Inno- vation Directorate A - Policy Development and Coordination Unit A1 Internal and external communication. Online verfügbar unter http://www.openaccess.gr/sites/openaccess.gr/files/Openinnovation.pdf, zuletzt geprüft am 09.06.2017.

Fecher, Benedikt; Friesike, Sascha (2014): Open Science: One Term, Five Schools of Thought. In: Sönke Bartling und Sascha Friesike (Hg.): Opening Science. The Evolving Guide on How the Internet is Changing Research, Collaboration and Scholarly Publishing. Cham: Springer, S. 17-47.

Franzen, Martina (2016): Open Science als wissenschaftspolitische Problemlösungsformel? In: Dagmar Simon, Andreas Knie, Stefan Hornbostel und Karin Zimmermann (Hg.): Handbuch der Wissenschaftspolitik. Wiesbaden: Springer Fachmedien, S. 279-296.

Franzoni, Chiara; Sauermann, Henry (2014): Crowd Science: The Organization of Scientific Research in Open Collaborative Projects. In: Research Policy 43 (1), S. 1-20.

Friesike, Sascha; Widenmayer, Bastian; Gassmann, Oliver; Schildhauer, Thomas (2015): Opening Science: Towards an Agenda of Open Science in Academia and Industry. In: Journal of Technology Transfer 40 (4), S. 581-601. DOI: 10.1007/s10961-014-9375-6.

Groom, Quentin; Weatherdon, Lauren; Geijzendorffer, Ilse R. (2016): Is Citizen Science an Open Science in the Case of Biodiversity Observations? In: Journal of Applied Ecology 54 (2), S. 612-617. DOI: 10.1111/1365-2664.12767. Grossman, Robert L. et al. (2012): The Design of a Community Science Cloud: The Open Science Data Cloud Perspective. In: High Performance Computing, Networking, Storage and Analysis (SCC). Online verfügbar unter https://www.computer.org/csdl/proceedings/scc/2012/6218/00/06495909abs.html, zuletzt geprüft am 09.06.2017.

Holmberg, Kim Johan (2015): Altmetrics for Information Professionals. Past, Present and Future. Waltham, MA: Chandos Publishing.

Irwin, Alan (1995): Citizen Science. London: Routledge.

Kullenberg, Christopher; Kasperowski, Dick (2016): What Is Citizen Science? A Scientometric Meta-Analysis. In: PLOS ONE 11 (1): e0147152. Online verfügbar unter https://doi.org/10.1371/journal.pone.0147152, zuletzt geprüft am 09. 06.2017.

Luna, Soledad; Sturm, Ulrike (2017): Defining Principles and Guidelines for Mobile Apps and Platform Development for Best Practice in Citizen Science. Workshop Report. Online verfügbar unter https://ecsa.citizen-science.net/sites/ default/files/report_of_the_workshop.pdf, zuletzt geprüft am 09.06.2017.

Mahr, Dana; Goebel, Claudia; Irwin, Alan; Vohland, Katrin (im Druck): Citizen Science Studies. How to Co-produce Reflexiveness and Productive Dialogue between Citizen Science Practitioners and Researchers. In: Aletta Bonn et al. (Hg.): Citizen Science - Innovation in Open Science, Society and Policy, London: UCL Press.

Moedas, Carlos (2016): Foreword by Commissioner Carlos Moedas. In: European Commission, Directorate-General for Research and Innovation. Realising the European Open Science Cloud. S. 4. Online verfügbar unter https://ec.europa.eu/research/openscience/pdf/realising_the_european_ open_science_cloud_2016.pdf, zuletzt geprüft am 09.06.2017.

Nentwich, Michael; König, René (2012): Cyberscience 2.0 - Research in the Age of Digital Social Networks. Frankfurt am Main: Campus.

Newman, Greg; Wiggins, Andrea; Crall, Alycia; Graham, Eric; Newman, Sarah; Crowston, Kevin (2012): The Future of Citizen Science: Emerging Technologies and Shifting Paradigms. In: Frontiers in Ecology and Environment 10 (6), S. 298-304. 
Open Knowledge International, 2015: Open Definition 2.1. Online verfügbar unter http://opendefinition.org/od/2.1/en/, zuletzt geprüft am 09.06.2017.

Oxford Dictionary (2017): Citizen Science. Verfügbar unter https:// en.oxforddictionaries.com/definition/citizen_science, zuletzt geprüft am 09. 06.2017.

Pettibone, Lisa; Oswald, Kristin; Smolarski, René (Hg.) (2016): GEWISS Dialogforum: Bürger Künste Wissenschaft. Berlin: Projekt „Bürger schaffen Wissen Wissen schafft Bürger" (GEWISS Bericht Nr. 8.). Online verfügbar unter http://www.buergerschaffenwissen.de/sites/default/files/assets/ dokumente/8_gewiss_bkw_bericht.pdf, zuletzt geprüft am 09.06.2017. Pettibone, Lisa; Ziegler, David; Vohland, Katrin (in Vorbereitung): What „Citizen Science" Means to Citizens. Results of a Pilot Study in Germany.

Preston, Benjamin (2016): Public Lab Puts Eco Justice in the Hands of Citizen Scientists. Online verfügbar unter http://makezine.com/2016/02/09/publiclab-puts-eco-justice-in-the-hands-citizen-scientists/, zuletzt geprüft am 09.06.2017.

Sanz, Fermín Serrano; Holocher-Ertl, Teresa; Kieslinger, Barbara; García, Francisco Sanz; Silva, Cândida G. (2014): White Paper on Citizen Science for Europe. Online verfügbar unter http://www.ecsite.eu/sites/default/files/white_ paper-final-print.pdf, zuletzt geprüft am 09.06.2017.

Schade, Sven; Bowser, Anne; Scarpino, Russell (2016): Data and Metadata: Reporting from the Citizen Science Data and Service Infrastructure Meeting in Italy. Workshop Report, Citizen Science Association (CSA). Online verfügbar unter http://citizenscience.org/2016/02/09/data-and-metadata-reportingfrom-the-citizen-science-data-and-service-infrastructure-meeting-in-italy/, zuletzt geprüft 09.06.2017.

SCU - Science Communication Unit, University of the West of England, Bristol (2013): Science for Environment Policy. In-depth Report: Environmental Citizen Science. Report produced for the European Commission DG Environment, December 2013. Online verfügbar unter http://ec.europa.eu/ environment/integration/research/newsalert/pdf/IR9_en.pdf, zuletzt geprüft am 09. 06.2017.

Shirk, Jennifer; Bonney, Rick (2015): Developing a Citizen Science Program: A Synthesis of Citizen Science Frameworks. Ithaca, NY: Cornell Lab of
Ornithology. Online verfügbar unter https://cdn1.safmc.net/wp-content/ uploads/2016/11/28101104/CS1_Developing20a20Citizen20Science20Program2 OGUIDE.pdf, zuletzt geprüft am 09.06.2017.

Smith, Elta; Gunashekar, Salil; Lichten, Catherine; Parks, Sarah; Chataway, Joanna (2016): A Framework to Monitor Open Science Trends in the EU. Online verfügbar unter https://www.oecd.org/sti/063\%20-\%200ECD\%20Blue\%20Sky\%20 2016_Open\%20Science.pdf, zuletzt geprüft am 09.06.2017.

Trojok, Rüdiger (2016): Biohacking. Gentechnologie für alle. München: Franzis verlag.

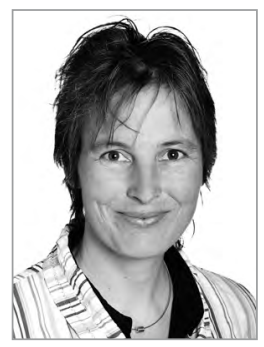

\section{DR. KATRIN VOHLAND}

leitet den Forschungsbereich „Wissenschaftskommunikation und Wissensforschung " am Museum für Naturkunde Berlin, Leibniz Institut für Evolutionsund Biodiversitätsforschung. Sie forscht zur Schnittstelle zwischen Wissenschaft, Politik und Gesellschaft. Sie koordiniert eine Cost Action zu Citizen Science und ist Vize-Chair von ECSA.

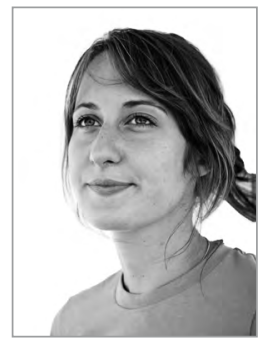

\section{CLAUDIA GÖBEL}

ist Projektleiterin bei der European Citizen Science Association (ECSA) und Gastwissenschaftlerin am Museum für Naturkunde Berlin. Mit akademischem Hintergrund in Wissenschafts- und Technikforschung arbeitet und forscht sie zu Citizen Science im Hinblick auf Organisationsstrukturen, Forschungsdaten und politische Relevanz.

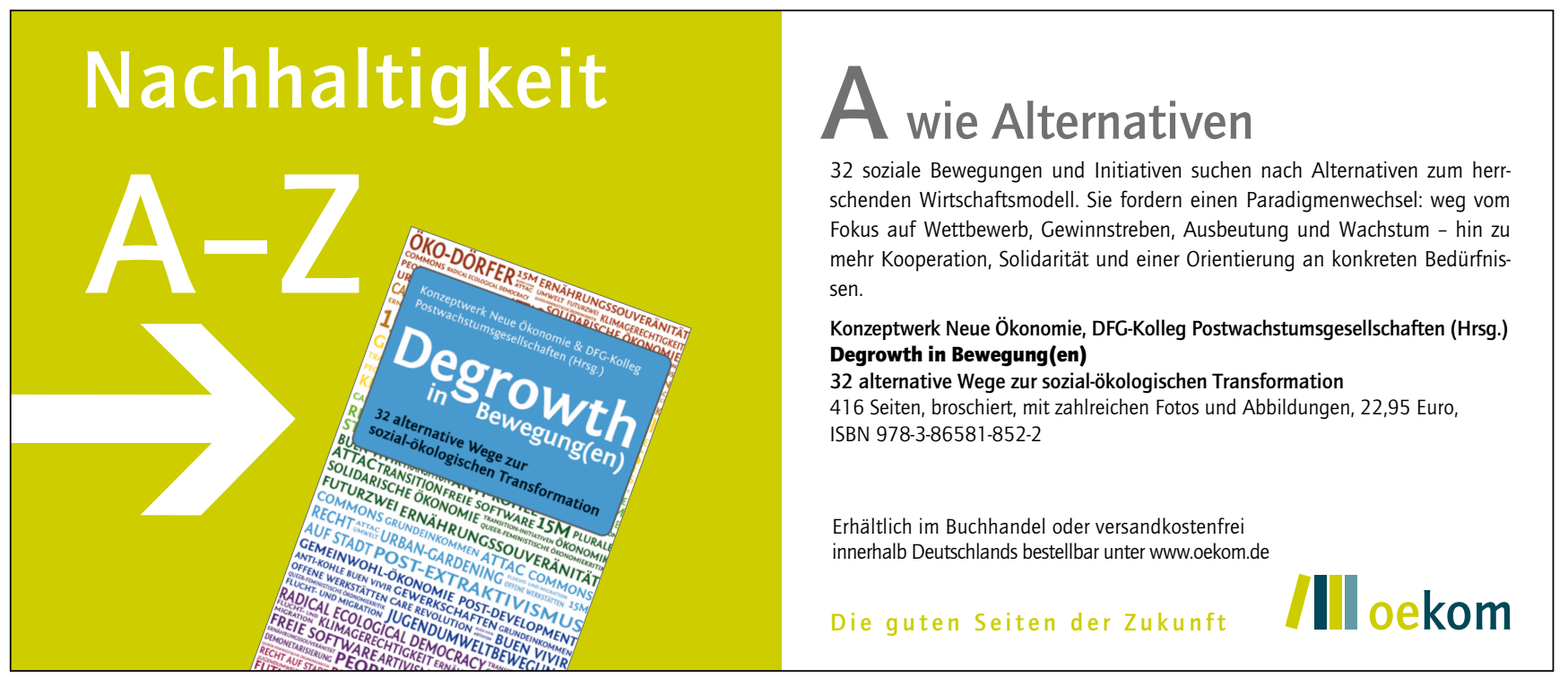

